Ann. Funct. Anal. 6 (2015), no. 3, 275-295

http://doi.org/10.15352/afa/06-3-20

ISSN: 2008-8752 (electronic)

http://projecteuclid.org/afa

\title{
ADVANCES IN OPERATOR CAUCHY-SCHWARZ INEQUALITIES AND THEIR REVERSES
}

\author{
J. M. ALDAZ ${ }^{1}$, S. BARZA ${ }^{2 *}$, M. FUJII ${ }^{3}$, M. S. MOSLEHIAN ${ }^{4}$ \\ Communicated by T. Yamazaki
}

\begin{abstract}
The Cauchy-Schwarz (C-S) inequality is one of the most famous inequalities in mathematics. In this survey article, we first give a brief history of the inequality. Afterward, we present the C-S inequality for inner product spaces. Focusing on operator inequalities, we then review some significant recent developments of the C-S inequality and its reverses for Hilbert space operators and elements of Hilbert $C^{*}$-modules. In particular, we pay special attention to an operator Wielandt inequality.
\end{abstract}

\section{INTRODUCTION}

One of the fundamental inequalities in mathematics is the Cauchy-Schwarz (C-S) inequality, which is known in the literature also as the Cauchy inequality, the Schwarz inequality or the Cauchy-Bunyakovsky-Schwarz inequality. Its most familiar version states that in a semi-inner product space $(\mathscr{X},\langle\cdot, \cdot\rangle)$, it holds

$$
|\langle x, y\rangle| \leq\|x\|\|y\| \quad(x, y \in \mathscr{X})
$$

Date: Received: Feb. 15, 2015; Accepted: Apr. 1, 2015.

* Corresponding author.

2010 Mathematics Subject Classification. Primary 47A63; Secondary 46L05, 47A30, 26D15.

Key words and phrases. History of mathematics, Operator inequality, operator geometric mean, CauchySchwarz inequality. 
where $\|x\|:=\langle x, x\rangle^{1 / 2}$. Equality in (1.1) occurs if and only if any one of $x, y$ is a scalar multiple of the other. Inequality (1.1) is equivalent to the positive semi-definiteness of the Gram matrix $\left[\begin{array}{cc}\langle x, x\rangle & \langle x, y\rangle \\ \langle y, x\rangle & \langle y, y\rangle\end{array}\right]$.

Let us have a look at its historical origin. In 1821, Augustin-Louis Cauchy [12] established the inequality for sums, namely

$$
\left(\sum_{i=1}^{n} a_{i} b_{i}\right)^{2} \leq \sum_{i=1}^{n} a_{i}^{2} \sum_{i=1}^{n} b_{i}^{2} \quad\left(a_{i}, b_{i} \in \mathbb{R}\right) .
$$

In 1859, Viktor Bunyakovsky [10], who was a student of Cauchy, gave a version for integrals in the form

$$
\left|\int_{a}^{b} f(t) \overline{g(t)} d t\right|^{2} \leq \int_{a}^{b}|f(t)|^{2} d t \int_{a}^{b}|g(t)|^{2} d t \quad\left(f, g \in \mathcal{L}^{2}([a, b])\right),
$$

with equality when there exist constants $\alpha, \beta$ not both equal to zero such that $\alpha \int_{a}^{s} f(t) d t=$ $\beta \int_{a}^{s} g(t) d t$ for all $s \in[a, b]$. The general form of the C-S inequality for inner product spaces was proved by Hermann Amandus Schwarz in 1885; see also [45].

The C-S inequality is a very important inequality with many elegant applications, for instance, in

- Classical and modern analysis

The C-S inequality is used

(i) to show the triangle inequality for $\|x\|:=\langle x, x\rangle^{1 / 2}$;

(ii) to prove the continuity of the inner product $\langle\cdot, \cdot\rangle$;

(iii) to establish the Bessel inequality;

(iv) to extend the notion of "angle $\theta_{x, y}$ between two vectors $x, y$ in the Euclidean plane" to any real inner product space by $\cos \theta_{x, y}:=\frac{\langle x, y\rangle}{\|x\|\|y\|}$;

(v) to prove some classical inequalities. For example, in order to prove that if $a_{1}, \cdots, a_{n}$ are non-negative real numbers such that $a_{1}+\cdots+a_{n} \leq n$, then $\frac{1}{a_{1}}+$ $\cdots+\frac{1}{a_{n}} \geq n$, it is enough to put $x_{i}=\sqrt{a_{i}}$ and $y_{i}=1 / \sqrt{a_{i}}$ in the C-S inequality (1.1).

- Partial differential equations

One may seek some inequalities, which relates norms of functions to norms of their derivatives

- Multivariable calculus

Using the C-S inequality we have $\left|D_{u}(f)\right| \leq|\nabla f||u|$, where $D_{u}(f)$ denotes the directional derivative of $f$ in the direction $u$ and $\nabla f$ is the gradient vector of $f$. 
- Probability theory

The variance-covariance inequality $\operatorname{cov}(X, Y) \leq \operatorname{var}(X) \operatorname{var}(Y)$ for random variables $X$ and $Y$ is a consequence of the C-S inequality.

- Physics

Schrödinger derived the so-called Schrödinger uncertainity relation from the C-S inequality and then obtained the Heisenberg uncertainty relation $\sigma_{x}^{2} \sigma_{y}^{2} \geq \hbar^{2} / 4$ in the Hilbert space of quantum observables as a special case.

\section{C-S INEQUALity IN Classical ANALYSiS}

For real inner product spaces, there are some elegant proofs of the C-S inequality. Assume that $\|x\|=\|y\|=1$. Then, the fact that $0 \leq\langle x-y, x-y\rangle=\langle x, x\rangle-2\langle x, y\rangle+\langle y, y\rangle$ implies that $\langle x, y\rangle \leq 1=\|x\|\|y\|$.

A similar argument can be used to derive the C-S inequality from the parallelogram identity

$$
\|x+y\|^{2}+\|x-y\|^{2}=2\|x\|^{2}+2\|y\|^{2} .
$$

This was noticed in [1] for real inner product spaces, with the modifications in the complex case appearing in the latter paper [2]. In the real case, for non-zero vectors $x$ and $y$, the parallelogram identity can simply be rewritten (we give the details in the proof of the next theorem) as

$$
\langle x, y\rangle=\|x\|\|y\|\left(1-\frac{1}{2}\left\|\frac{x}{\|x\|}-\frac{y}{\|y\|}\right\|^{2}\right) .
$$

Thus, the size of $\langle x, y\rangle$ is determined by the angular distance $\left\|\frac{x}{\|x\|}-\frac{y}{\|y\|}\right\|$ between $x$ and $y$. In particular, $\langle x, y\rangle \leq\|x\|\|y\|$, with equality precisely when the angular distance is zero.

In what follows it is convenient to replace the nonzero vectors $x$ and $y$ by unit vectors $u=x /\|x\|$ and $v=y /\|y\|$.

Theorem 2.1. For all nonzero vectors $x$ and $y$ in a complex inner product space,

$$
\operatorname{Re}\langle x, y\rangle=\|x\|\|y\|\left(1-\frac{1}{2}\left\|\frac{x}{\|x\|}-\frac{y}{\|y\|}\right\|^{2}\right)
$$

and

$$
\operatorname{Im}\langle x, y\rangle=\|x\|\|y\|\left(1-\frac{1}{2}\left\|\frac{x}{\|x\|}-\frac{i y}{\|y\|}\right\|^{2}\right)
$$


Proof. Let $\|u\|=\|v\|=1$. From (2.1) we obtain

$$
4-\|u-v\|^{2}=\|u+v\|^{2}=2+\langle u, v\rangle+\langle v, u\rangle=2+\langle u, v\rangle+\overline{\langle u, v\rangle}=2+2 \operatorname{Re}\langle u, v\rangle .
$$

Thus, $\operatorname{Re}\langle u, v\rangle=1-\frac{1}{2}\|u-v\|^{2}$. The same argument, applied to $\|u+i v\|^{2}$, yields $\operatorname{Im}\langle u, v\rangle=$ $1-\frac{1}{2}\|u-i v\|^{2}$.

Let $\operatorname{Arg} z$ denote the principal argument of $z \in \mathbb{C}, z \neq 0$. That is, $-\pi<\operatorname{Arg} z \leq \pi$, and in polar coordinates, $z=e^{i \operatorname{Arg} z} r$, where $r=|z|$.

Theorem 2.2. Let $x$ and $y$ be nonzero vectors in a complex inner product space. Then

$$
|\langle x, y\rangle|=\|x\|\|y\|\left(1-\frac{1}{2}\left\|\frac{e^{-i \operatorname{Arg}\langle x, y\rangle} x}{\|x\|}-\frac{y}{\|y\|}\right\|^{2}\right) .
$$

Proof. By a normalization, it is enough to consider unit vectors $u$ and $v$. Set $t=\operatorname{Arg}\langle u, v\rangle$, so $\langle u, v\rangle=e^{i t} r$ in polar form. Using (2.3) we obtain

$$
|\langle u, v\rangle|=r=\left\langle e^{-i t} u, v\right\rangle=\operatorname{Re}\left\langle e^{-i t} u, v\right\rangle=1-\frac{1}{2}\left\|e^{-i t} u-v\right\|^{2} .
$$

And now C-S inequality follows, with equality for nonzero $x, y$ precisely when one of the vectors is a scalar multiple of the other, that is, when for some $\alpha \in \mathbb{R}, \frac{e^{i \alpha} x}{\|x\|}=\frac{y}{\|y\|}$.

There are also several proofs "without words". Among them we mention the following interesting one for (1.2) due to Nelsen [40]:
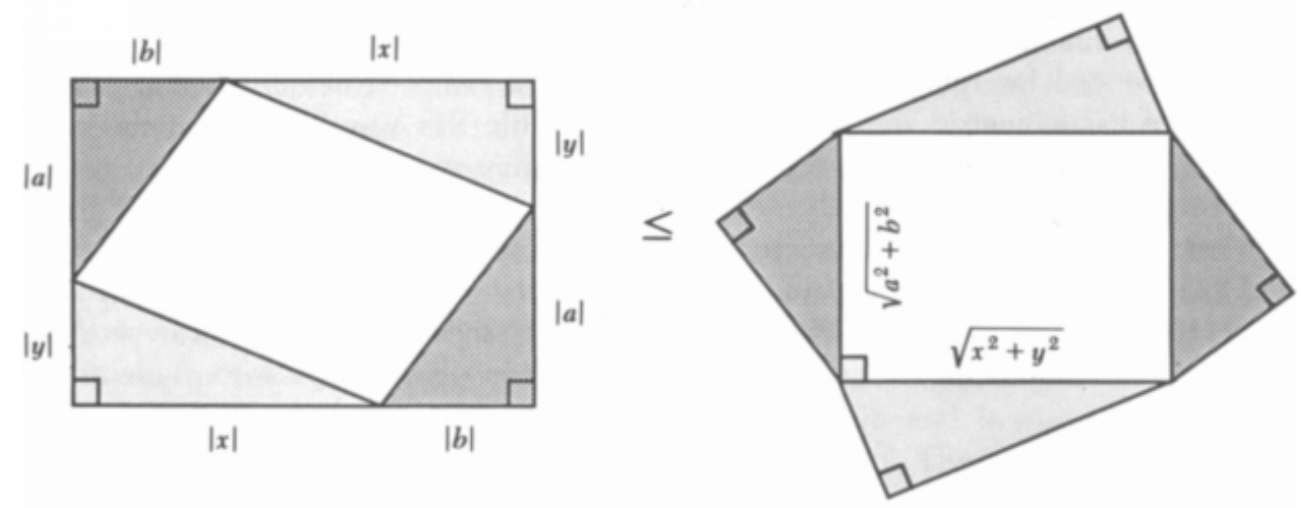

$$
\begin{gathered}
(|a|+|y|)(|b|+|x|) \leq 2\left(\frac{1}{2}|a||b|+\frac{1}{2}|x||y|\right)+\sqrt{a^{2}+b^{2}} \sqrt{x^{2}+y^{2}} \\
\therefore|a x+b y| \leq|a||x|+|b||y| \leq \sqrt{a^{2}+b^{2}} \sqrt{x^{2}+y^{2}}
\end{gathered}
$$


There are some inequalities equivalent to the C-S inequality. One of them is the Wagner inequality which follows by employing the C-S inequality (1.1) to the following semi-inner product

$$
[f, g]:=\int_{\Omega} \operatorname{Re}\langle f(t), g(t)\rangle d \mu+\alpha \iint_{\Omega \times \Omega-\Delta(\Omega \times \Omega)} \operatorname{Re}\langle f(t), g(s)\rangle d(\mu \times \mu) .
$$

Theorem 2.3. [17] Suppose that $(\Omega, \mu)$ is a measure space, $f, g$ are Bochner integrable Hilbert space-valued functions on $\Omega$ and $\alpha \in[0,1]$. Then

$$
\begin{aligned}
\left(\int_{\Omega} \operatorname{Re}\langle f(t), g(t)\rangle d \mu\right. & \left.\alpha \iint_{\Omega \times \Omega-\Delta(\Omega \times \Omega)} \operatorname{Re}\langle f(t), g(s)\rangle d(\mu \times \mu)\right)^{2} \\
\leq & \left(\int_{\Omega}\|f(t)\|^{2} d \mu+\alpha \iint_{\Omega \times \Omega-\Delta(\Omega \times \Omega)} \operatorname{Re}\langle f(t), f(s)\rangle d(\mu \times \mu)\right) \\
& \times\left(\int_{\Omega}\|g(t)\|^{2} d \mu+\alpha \iint_{\Omega \times \Omega-\Delta(\Omega \times \Omega)} \operatorname{Re}\langle g(t), g(s)\rangle d(\mu \times \mu)\right) .
\end{aligned}
$$

If $\Omega=\{1, \cdots, n\}, \mu(\{i\})=1, f(i)=a_{i} \in \mathbb{R}, g(i)=b_{i} \in \mathbb{R}$, then we get the following classical Wagner inequality:

Corollary 2.4. [48] Let $a_{1}, \ldots, a_{n}$ and $b_{1}, \ldots, b_{n}$ be real numbers. Then

$$
\left(\sum_{i=1}^{n} a_{i} b_{i}+\alpha \sum_{1 \leq i \neq j \leq n} a_{i} b_{j}\right)^{2} \leq\left(\sum_{i=1}^{n} a_{i}^{2}+\alpha \sum_{1 \leq i \neq j \leq n} a_{i} a_{j}\right)\left(\sum_{i=1}^{n} b_{i}^{2}+\alpha \sum_{1 \leq i \neq j \leq n} b_{i} b_{j}\right)
$$

Let $(\Omega, \mu)$ be a measure space, $\rho: \Omega \rightarrow[0, \infty)$ be a measurable function and

$$
\mathcal{L}_{\rho}^{2}(\Omega, \mu):=\left\{f: \Omega \rightarrow \mathbb{C} \mid f \text { is measurable and } \int_{\Omega} \rho(t)|f(t)|^{2} d \mu(t)<\infty\right\},
$$

which is a Hilbert space equipped with the natural inner product $\langle f, g\rangle=\int_{\Omega} \rho f \bar{g} d \mu \quad(f, g \in$ $\left.\mathcal{L}_{\rho}^{2}(\Omega, \mu)\right)$. From Theorem 2.3 we get now the following corollary.

Corollary 2.5. Let $(\Omega, \mu)$ be a positive measure space, $\rho: \Omega \rightarrow[0, \infty)$ be a measurable function and $f_{1}, \cdots, f_{n}, g_{1}, \cdots, g_{n}$ be real-valued functions of $\mathcal{L}_{\rho}^{2}(\Omega, \mu)$. Then

$$
\begin{aligned}
\left(\sum_{i=1}^{n} \int_{\Omega} \rho(t) f_{i}(t) g_{i}(t) d \mu(t)+\right. & \left.\alpha \sum_{1 \leq i \neq j \leq n} \int_{\Omega} \rho(t) f_{i}(t) g_{j}(t) d \mu(t)\right)^{2} \\
\leq & \left(\sum_{i=1}^{n} \int_{\Omega} \rho(t)\left|f_{i}(t)\right|^{2} d \mu(t)+2 \alpha \sum_{1 \leq i<j \leq n} \int_{\Omega} \rho(t) f_{i}(t) f_{j}(t) d \mu(t)\right) \\
& \times\left(\sum_{i=1}^{n} \int_{\Omega} \rho(t)\left|g_{i}(t)\right|^{2} d \mu(t)+2 \alpha \sum_{1 \leq i<j \leq n} \int_{\Omega} \rho(t) g_{i}(t) g_{j}(t) d \mu(t)\right) .
\end{aligned}
$$


Several mathematicians generalized the C-S inequality in different ways; see [16]. For instance, Buzano [11] showed that $|\langle x, z\rangle\langle z, y\rangle| \leq \frac{1}{2}(\|x\|\|y\|+|\langle x, y\rangle|) \cdot\|z\|^{2}$ for three elements $x, y, z$ in a real or complex Hilbert space. In addition, Alzer [3] proved that the inequality

$$
\left(\sum_{k=1}^{n} a_{k} b_{k}\right)^{2} \leq \sum_{k=1}^{n} b_{k} \sum_{k=1}^{n}\left(\alpha+\frac{\beta}{k}\right) a_{k}^{2} b_{k}
$$

holds for all natural numbers $n$ and for all real numbers $a_{k}$ and $b_{k}(k=1, \cdots, n)$ with $0<a_{1} \leq a_{2} / 2 \leq \cdots \leq a_{n} / n$ and $0<b_{n} \leq b_{n-1} \leq \cdots \leq b_{1}$, if and only if $\alpha \geq 3 / 4$ and $\beta \geq 1-\alpha$.

\section{Operator versions of the C-S inequality}

Let $\mathbb{B}(\mathscr{H})$ denote the $C^{*}$-algebra of all bounded linear operators on a complex Hilbert space $(\mathscr{H},\langle\cdot, \cdot\rangle)$ equipped with the operator norm and the adjoint operation $A \mapsto A^{*}$ via $\langle A x, y\rangle=\left\langle x, A^{*} y\right\rangle$. From now on, a capital letter denotes an operator in $\mathbb{B}(\mathscr{H})$. If $\operatorname{dim} \mathscr{H}=$ $n$, then $\mathbb{B}(\mathscr{H})$ can be identified with the space $\mathbb{M}_{n}$ of all $n \times n$ complex matrices. We identify a scalar with the identity operator $I$ multiplied by this scalar. An operator $A \in \mathbb{B}(\mathscr{H})$ is called self-adjoint if $A^{*}=A$.

For self-adjoint operators $A, B \in \mathbb{B}(\mathscr{H})$ the partially ordered relation $B \leq A$ means that $\langle B x, x\rangle \leq\langle A x, x\rangle$ for all $x \in \mathscr{H}$. In particular, if $A \geq 0$, then $A$ is called positive. If $A$ is a positive invertible operator, then we write $A>0$. A map $\Phi: \mathscr{A} \rightarrow \mathscr{B}$ between two $C^{*}$-algebras is said to be positive if $\Phi(A) \geq 0$ whenever $A \geq 0$. It is called $n$-positive if $\Phi \otimes I_{n}: M_{n}(\mathscr{A}) \rightarrow M_{n}(\mathscr{B})$ is positive, where $M_{n}(\mathscr{A})$ is the $C^{*}$-algebra of $n \times n$ matrices with entries in $\mathscr{A}$ and $I_{n}$ denotes its identity matrix. We say that $\Phi$ is completely positive if it is $n$-positive for all $n$. If $\Phi$ preserves the identity, then it is called unital. The reader is referred to [26] for undefined notations and terminologies.

Let $\Phi: \mathscr{A} \rightarrow \mathscr{B}$ be a unital positive linear map between $C^{*}$-algebras. Kadison [31] generalized the C-S inequality by showing that $\Phi\left(A^{2}\right) \geq \Phi(A)^{2}$ for every self-adjoint operator $A$ in $\mathscr{A}$. Choi [14] extended the result of Kadison. To establish Choi's result we need the following two theorems:

Theorem 3.1. [46] If $\Phi: \mathscr{A} \rightarrow \mathscr{B}$ is a unital positive linear map and $\mathscr{A}$ is commutative, then $\Phi$ is completely positive.

Theorem 3.2 (Stinespring Theorem). [46] Suppose that $\Phi$ is a completely positive unital map from a $C^{*}$-algebra $\mathscr{A}$ into $\mathbb{B}(\mathscr{K})$. Then there exist a representation $\pi$ of $\mathscr{A}$ on a Hilbert space $\mathscr{H}$ and an isometry $V$ from $\mathscr{H}$ into $\mathscr{K}$ such that $\Phi(X)=V^{*} \pi(X) V$ for all $X \in \mathscr{A}$. 
Theorem 3.3 (Choi inequality). [14] Suppose that $\Phi: \mathscr{A} \rightarrow \mathscr{B}$ is a unital positive linear map. Then

$$
\Phi\left(A^{*} A\right) \geq \Phi(A)^{*} \Phi(A)
$$

for all normal operators $A \in \mathbb{B}(\mathscr{H})$.

Proof. Let $C^{*}(A, I)$ denote the commutative $C^{*}$-algebra generated by $A$ and $I$. The restriction of $\Phi$ to $C^{*}(A, I)$ is completely positive. Hence, by the Stinespring Theorem, it admits a decomposition of the form $\Phi(X)=V^{*} \pi(X) V\left(X \in C^{*}(A, I)\right)$, where $\pi$ is a representation of $C^{*}(A, I)$ on a Hilbert space $\mathscr{L}$ and $V$ is an isometry from $\mathscr{L}$ into $\mathscr{K}$. We have

$$
\Phi(A)^{*} \Phi(A)=V^{*} \pi\left(A^{*}\right) V V^{*} \pi(A) V \leq V^{*} \pi\left(A^{*}\right) \pi(A) V=V^{*} \pi\left(A^{*} A\right) V=\Phi\left(A^{*} A\right),
$$

since $V^{*} V=I$. Therefore $\left\|V V^{*}\right\|=\left\|V^{*} V\right\|=1$ and hence $V V^{*} \leq I$.

If $\Phi$ is a completely positive map on $\mathbb{B}(\mathscr{H})$, then the covariance between any two operators is defined by $\operatorname{cov}(A, B)=\phi\left(A^{*} B\right)-\phi(A)^{*} \phi(B)$. Bhatia and Davis [9] generalized Kadison's Schwarz inequality by showing that for any operators $A_{1}, \ldots, A_{n}$, the block matrix $\left[\operatorname{cov}\left(A_{i}, A_{j}\right)\right]$ is positive. Mathias [37] proved that for any $(n+1)$-positive map $\Phi$ and any bounded linear operators $A_{i}, i=1, \ldots, n$, it holds that $\left[\Phi\left(A_{i}^{*} A_{j}\right)\right]_{i, j=1}^{n} \geq\left[\Phi\left(A_{i}\right)^{*} \Phi\left(A_{j}\right)\right]_{i, j=1}^{n}$ and showed that if $(n+1)$-positive is replaced by $n$-positive, then the statement is not valid in general. An application of the covariance-variance inequality to the C-S inequality was obtained by M. Fujii et al. [22].

For positive operators $\left\{A_{i}\right\}_{i=1}^{m}$ and $\left\{B_{i}\right\}_{i=1}^{m}$ in $\mathbb{B}(\mathscr{H})$, the inequality

$$
\sum_{i=1}^{m} A_{i} \sharp B_{i} \leq\left(\sum_{i=1}^{m} A_{i}\right) \sharp\left(\sum_{i=1}^{m} B_{i}\right),
$$

which is equivalent to the concavity of the operator geometric mean $\sharp$, defined by $A \sharp B:=$ $A^{1 / 2}\left(A^{-1 / 2} B A^{-1 / 2}\right)^{1 / 2} A^{1 / 2}$, is an operator C-S type inequality; see, e.g, [26, Chapter V]. Furthermore, some C-S inequalities for Hilbert space operators and matrices involving unitarily invariant norms were given by Jocić [29] and Kittaneh [32]. A refinement of the C-S inequality involving operator means is investigated by Wada [47]. Some operator versions of the C-S inequality with simple conditions for the case of equality are presented by Fujii [19].

In addition, there are some generalization of the C-S inequality for matrices and unitarily invariant norms. For instance, Bhatia and Davis [8] proved that

$$
\left.\left\|\left.|| A^{*} X B\right|^{r}||^{2} \leq\right\||| A A^{*} X\right|^{r}\left|\left\|\left.|\cdot||| X B B^{*}\right|^{r}\right\| \|,\right.
$$

holds for all $A, B, X \in M_{n}$ and any real number $r>0$. 


\section{C-S inequality And its Reverse in Hilbert $C^{*}$-MOdules}

The notion of semi-inner product $C^{*}$-module is a natural generalization of that of semiinner product space arising under replacement of the field of scalars $\mathbb{C}$ by a $C^{*}$-algebra. Let $\mathscr{A}$ be a $C^{*}$-algebra and let $\mathscr{X}$ be an algebraic right $\mathscr{A}$-module which is a complex linear space with $(\lambda x) A=x(\lambda A)=\lambda(x A)$ for all $x \in \mathscr{X}, A \in \mathscr{A}, \lambda \in \mathbb{C}$. The space $\mathscr{X}$ is said to be a (right) semi-inner product $\mathscr{A}$-module if there exists an $\mathscr{A}$-valued inner product, i.e., a mapping $\langle\cdot, \cdot\rangle: \mathscr{X} \times \mathscr{X} \rightarrow \mathscr{A}$ satisfying

(i) $\langle x, x\rangle \geq 0$, where " $\geq$ " denotes the usual order in the real space of self-adjoint elements of $\mathscr{A}$;

(ii) $\langle x, \lambda y+z\rangle=\lambda\langle x, y\rangle+\langle x, z\rangle$;

(iii) $\langle x, y A\rangle=\langle x, y\rangle$;

(iv) $\langle x, y\rangle^{*}=\langle y, x\rangle$.

for all $x, y, z \in \mathscr{X}, A \in \mathscr{A}, \lambda \in \mathbb{C}$. Moreover, if

(v) $x=0$ whenever $\langle x, x\rangle=0$,

then $\mathscr{X}$ is called an inner product $C^{*}$-module over the $C^{*}$-algebra $\mathscr{A}$. Clearly, every inner product space is an inner product $\mathbb{C}$-module. One can define a norm on $\mathscr{X}$ by $\|x\|=$ $\|\langle x, x\rangle\|^{\frac{1}{2}}$, where the latter norm is the norm in the $C^{*}$-algebra $\mathscr{A}$. If this normed space is complete, then $\mathscr{X}$ is called a Hilbert $\mathscr{A}$-module. A left inner product $\mathscr{A}$-module can be defined analogously. Any $C^{*}$-algebra $\mathscr{A}$ under $\langle A, B\rangle:=A^{*} B(A, B \in \mathscr{A})$ can be regarded as a right Hilbert $C^{*}$-module over itself.

Let $\mathscr{A}$ be a $C^{*}$-algebra with center $\mathcal{Z}(\mathscr{A})=\{A \in \mathscr{A}: A B=B A$ for all $B \in \mathscr{A}\}$ and let $(\mathscr{X},\langle\cdot, \cdot\rangle)$ be a semi-inner product $\mathscr{A}$-module. The following C-S inequality, whose proof is analogue to that of the classical one, is known [34]

$$
\langle x, y\rangle^{*}\langle x, y\rangle \leq\|\langle x, x\rangle\|\langle y, y\rangle \quad(x, y \in \mathscr{X}) .
$$

Ilišević and Varošanec [28] improved this inequality by showing that if $x, y \in \mathscr{X}$ and $\langle x, x\rangle \in$ $\mathcal{Z}(\mathscr{A})$, then

$$
\langle x, y\rangle^{*}\langle x, y\rangle \leq\langle x, x\rangle\langle y, y\rangle .
$$

Another version of the C-S inequality is presented in [20], in which the authors assume the invertibility of $\langle y, y\rangle$ instead of $\langle x, x\rangle \in \mathcal{Z}(\mathscr{A})$. More precisely, they showed that if $\mathscr{X}$ is a semi-inner product $C^{*}$-module over $\mathscr{A}$ and $x, y \in \mathscr{X}$ such that $\langle y, y\rangle$ is invertible, then

$$
\langle x, y\rangle\langle y, y\rangle^{-1}\langle x, y\rangle^{*} \leq\langle x, x\rangle .
$$


Ma [36] proved that

$$
\left|\|z\|^{2}\langle x, y\rangle-\langle x, z\rangle\langle y, z\rangle\right|^{2} \leq\left(\|z\|^{2}\|x\|^{2}-\langle x, z\rangle^{2}\right)\left(\|z\|^{2}\|y\|^{2}-\langle y, z\rangle^{2}\right)
$$

for $x, y, z$ in a real inner product space $(\mathscr{H},\langle\cdot, \cdot\rangle)$, which is nothing else than the C-S inequality for the semi-inner product $\langle x, y\rangle_{z}:=\|z\|^{2}\langle x, y\rangle-\langle x, z\rangle\langle z, y\rangle$. Arambasić et al. [5] showed the following C-S inequality for the semi-inner product $\langle\cdot, \cdot\rangle_{z}$ on a semi-inner product module $\mathscr{X}$ :

$$
\begin{array}{r}
\left(\|z\|^{2}\langle y, x\rangle-\langle y, z\rangle\langle z, x\rangle\right)\left(\|z\|^{2}\langle x, y\rangle-\langle x, z\rangle\langle z, y\rangle\right) \\
\leq\|\| z\left\|^{2}\langle x, x\rangle-\langle x, z\rangle\langle z, x\rangle\right\|\left(\|z\|^{2}\langle y, y\rangle-\langle y, z\rangle\langle z, y\rangle\right),
\end{array}
$$

which generalizes the result of [36]. In particular, if $\langle x, z\rangle=0$, then

$$
|\langle z, y\rangle|^{2} \leq \frac{\|z\|^{2}}{\|x\|^{2}}\left(\|x\|^{2}|y|^{2}-|\langle x, y\rangle|^{2}\right),
$$

which presents an Ostrowski type inequality in a semi-inner product $C^{*}$-module.

The next result is a generalization of both Klamkin-Mclenaghan's inequality and ShishaMond's inequality, see also [18, Theorem 2]. To prove it we need the following lemma.

Lemma 4.1. [20] Let $\mathscr{X}$ be a semi-inner product $C^{*}$-module over $\mathscr{A}$. Suppose that $x, y \in \mathscr{X}$ such that $\langle x, y\rangle$ is normal and

$$
\operatorname{Re}\langle A y-x, x-B y\rangle \geq 0
$$

for some $A, B \in \mathcal{Z}(\mathscr{A})$. Then

$$
\langle x, x\rangle+\operatorname{Re}\left(A B^{*}\right)\langle y, y\rangle \leq|B+A||\langle x, y\rangle|
$$

where $|A|$ denotes the positive square root of the positive operator $A^{*} A$ for $A \in \mathscr{A}$ and $\operatorname{Re} A=\left(A+A^{*}\right) / 2$ is the real part of $A$.

Theorem 4.2. [20] Let $\mathscr{X}$ be a semi-inner product $C^{*}$-module over $\mathscr{A}$. Suppose that $x, y \in$ $\mathscr{X}$ such that $\langle x, y\rangle$ is normal and invertible, $\langle y, y\rangle$ is invertible and $A, B \in \mathcal{Z}(\mathscr{A})$ satisfy $\operatorname{Re}\left(A B^{*}\right) \geq 0$ and (4.3). Then

$$
|\langle x, y\rangle|^{-\frac{1}{2}}\langle x, x\rangle|\langle x, y\rangle|^{-\frac{1}{2}}-|\langle x, y\rangle|^{\frac{1}{2}}\langle y, y\rangle^{-1}|\langle x, y\rangle|^{\frac{1}{2}} \leq|A+B|-2 \operatorname{Re}\left(A B^{*}\right)^{\frac{1}{2}} .
$$


Proof. Employing Lemma 4.1 we get

$$
\begin{aligned}
& |\langle x, y\rangle|^{-\frac{1}{2}}\langle x, x\rangle|\langle x, y\rangle|^{-\frac{1}{2}}-|\langle x, y\rangle|^{\frac{1}{2}}\langle y, y\rangle^{-1}|\langle x, y\rangle|^{\frac{1}{2}} \\
& \leq|A+B|-\operatorname{Re}\left(A B^{*}\right)|\langle x, y\rangle|^{-\frac{1}{2}}\langle y, y\rangle|\langle x, y\rangle|^{-\frac{1}{2}}-|\langle x, y\rangle|^{\frac{1}{2}}\langle y, y\rangle^{-1}|\langle x, y\rangle|^{\frac{1}{2}} \\
& =|A+B|-2 \operatorname{Re}\left(A B^{*}\right)^{\frac{1}{2}} \\
& \quad-\left(\operatorname{Re}\left(A B^{*}\right)^{\frac{1}{2}}\left(|\langle x, y\rangle|^{-\frac{1}{2}}\langle y, y\rangle|\langle x, y\rangle|^{-\frac{1}{2}}\right)^{\frac{1}{2}}-\left(|\langle x, y\rangle|^{\frac{1}{2}}\langle y, y\rangle^{-1}|\langle x, y\rangle|^{\frac{1}{2}}\right)^{\frac{1}{2}}\right)^{2} \\
& \leq|A+B|-2 \operatorname{Re}\left(A B^{*}\right)^{\frac{1}{2}} .
\end{aligned}
$$

A weighted integral version of Klamkin-Mclenaghan's inequality reads as follows.

Corollary 4.3. Let $f, g \in \mathcal{L}_{\rho}^{2}(\Omega, \mu)$ be real functions such that $\int_{\Omega} \rho f g d \mu \neq 0, g \neq 0$ almost everywhere and $m g \leq f \leq M g$ for some scalars $M>m>0$. Then

$$
\frac{\int_{\Omega} \rho|f|^{2} d \mu}{\left|\int_{\Omega} \rho f g d \mu\right|}-\frac{\left|\int_{\Omega} \rho f g d \mu\right|}{\int_{\Omega} \rho|g|^{2} d \mu} \leq(\sqrt{M}-\sqrt{m})^{2} .
$$

Proof. Theorem 4.2 ensures the desired inequality since $\langle M g-f, f-m g\rangle \geq 0$.

The next result gives an additive reverse C-S inequality.

Theorem 4.4. [20] Let $\mathscr{X}$ be a semi-inner product $C^{*}$-module over $\mathscr{A}$. Suppose that $x, y \in$ $\mathscr{X}$ such that $\langle x, y\rangle$ is normal, and $A, B \in \mathcal{Z}(\mathscr{A}),|A+B|$ is invertible and (4.3) holds. Then

$$
\operatorname{Re}\left(\langle x, x\rangle^{\frac{1}{2}}\langle y, y\rangle^{\frac{1}{2}}\right)-|\langle x, y\rangle| \leq \frac{1}{4}|A-B|^{2}|A+B|^{-1}\langle y, y\rangle .
$$

Proof. It follows from Lemma 4.1 that

$$
\begin{aligned}
& \operatorname{Re}\left(\langle x, x\rangle^{\frac{1}{2}}\langle y, y\rangle^{\frac{1}{2}}\right)-|\langle x, y\rangle| \\
\leq & \operatorname{Re}\left(\langle x, x\rangle^{\frac{1}{2}}\langle y, y\rangle^{\frac{1}{2}}\right)-|A+B|^{-1}\langle x, x\rangle-|A+B|^{-1} \operatorname{Re}\left(A B^{*}\right)\langle y, y\rangle \\
= & {\left[\frac{1}{4}|A+B|-\operatorname{Re}\left(A B^{*}\right)|A+B|^{-1}\right]\langle y, y\rangle } \\
& \quad-|A+B|^{-1}\left(\langle x, x\rangle^{\frac{1}{2}}-\frac{1}{2}|A+B|\langle y, y\rangle^{\frac{1}{2}}\right)^{2} \\
\leq & \frac{1}{4}\left[|A+B|^{2}-4 \operatorname{Re}\left(A B^{*}\right)\right]|A+B|^{-1}\langle y, y\rangle \\
= & \frac{1}{4}|A-B|^{2}|A+B|^{-1}\langle y, y\rangle .
\end{aligned}
$$


Corollary 4.5. Let $\varphi$ be a positive linear functional on a $C^{*}$-algebra $\mathscr{A}$ and let $A, B \in \mathscr{A}$ be such that

$$
\operatorname{Re} \varphi\left((\Lambda B-A)^{*}(A-\lambda B)\right) \geq 0
$$

for some $\lambda, \Lambda \in \mathbb{C}$. Then

$$
\varphi\left(A^{*} A\right)^{1 / 2} \varphi\left(B^{*} B\right)^{1 / 2}-\left|\varphi\left(B^{*} A\right)\right| \leq \frac{|\Lambda-\lambda|^{2}}{4|\Lambda+\lambda|} \min \left\{\varphi\left(B^{*} B\right), \varphi\left(A^{*} A\right)\right\} .
$$

Proof. The $C^{*}$-algebra $\mathscr{A}$ can be regarded as a semi-inner product module over $\mathbb{C}$ via $\langle A, B\rangle=\varphi\left(B^{*} A\right)$. Now the required inequality follows from Theorem 4.4 and an obvious symmetry argument.

\section{Reverse C-S inequality in the Classical ANALysis}

Probably the first reverse C-S inequality for positive real numbers $a_{1}, \cdots, a_{n}$ is the following one due to G. Pólya and G. Szegö; see e.g. [42, p. 57 and 213-214]):

$$
\sum_{i=1}^{n} a_{i}^{2} \sum_{i=1}^{n} b_{i}^{2} \leq \frac{\left(m_{1} m_{2}+M_{1} M_{2}\right)^{2}}{4 m_{1} m_{2} M_{1} M_{2}}\left(\sum_{i=1}^{n} a_{i} b_{i}\right)^{2}
$$

where $0<m_{1} \leq a_{i} \leq M_{1}, 0<m_{2} \leq b_{i} \leq M_{2}(1 \leq i \leq n)$ for some constants $m_{1}, m_{2}, M_{1}, M_{2}$. The inequality is sharp in the sense that $1 / 4$ is the best possible constant. Another version of (5.1), which is a direct consequence of the arithmetic-geometric mean inequality reads as follows:

$$
\sum_{i=1}^{n} a_{i}^{2} \sum_{i=1}^{n} b_{i}^{2} \leq \frac{(M+m)^{2}}{4 M m}\left(\sum_{i=1}^{n} a_{i} b_{i}\right)^{2}
$$

whenever $0<m b_{i} \leq a_{i} \leq M b_{i}$. Equality holds if and only if there exist a permutation $\sigma$ of $\{1, \cdots, n\}$ and $0 \leq j \leq n$ such that $a_{\sigma(i)}=m b_{\sigma(i)}$ for $1 \leq \sigma(i) \leq j$ and $a_{\sigma(i)}=M b_{\sigma(i)}$ for $j+1 \leq \sigma(i) \leq n$ as well as $m \sum_{\sigma(i)=1}^{j} b_{\sigma(i)}^{2}=M \sum_{\sigma(i)=j+1}^{n} b_{\sigma(i)}^{2}$.

We remark that (5.1) can be rewritten in the following equivalent form

$$
\sum_{i=1}^{n} a_{i}^{2} \sum_{i=1}^{n} b_{i}^{2}-\left(\sum_{i=1}^{n} a_{i} b_{i}\right)^{2} \leq \frac{\left(M_{1} M_{2}-m_{1} m_{2}\right)^{2}}{4 m_{1} m_{2} M_{1} M_{2}}\left(\sum_{i=1}^{n} a_{i} b_{i}\right)^{2} .
$$

Inequality (5.1) is a multiplicative form and inequality (5.3) is an additive form of the reverse C-S inequality.

There are several reverse $\mathrm{C}-\mathrm{S}$ inequalities in the literature:

(i) If $\left(a_{1}, \ldots, a_{n}\right)$ and $\left(b_{1}, \ldots, b_{n}\right)$ are $n$-tuples of real numbers with $0<m_{1} \leq a_{i} \leq$ $M_{1}(1 \leq i \leq n), 0<m_{2} \leq b_{i} \leq M_{2}(1 \leq i \leq n)$, then 
- Diaz-Metcalf inequality [15]

$$
\sum_{k=1}^{n} b_{k}^{2}+\frac{m_{2} M_{2}}{m_{1} M_{1}} \sum_{k=1}^{n} a_{k}^{2} \leq\left(\frac{M_{2}}{m_{1}}+\frac{m_{2}}{M_{1}}\right) \sum_{k=1}^{n} a_{k} b_{k}
$$

- Pólya-Szegö inequality [42]

$$
\frac{\sum_{k=1}^{n} a_{k}^{2} \sum_{k=1}^{n} b_{k}^{2}}{\left(\sum_{k=1}^{n} a_{k} b_{k}\right)^{2}} \leq \frac{1}{4}\left(\sqrt{\frac{M_{1} M_{2}}{m_{1} m_{2}}}+\sqrt{\frac{m_{1} m_{2}}{M_{1} M_{2}}}\right)^{2}
$$

- Shisha-Mond inequality [44]

$$
\frac{\sum_{k=1}^{n} a_{k}^{2}}{\sum_{k=1}^{n} a_{k} b_{k}}-\frac{\sum_{k=1}^{n} a_{k} b_{k}}{\sum_{k=1}^{n} b_{k}^{2}} \leq\left(\sqrt{\frac{M_{1}}{m_{2}}}-\sqrt{\frac{m_{1}}{M_{2}}}\right)^{2} ;
$$

(ii) If $\left(a_{1}, \ldots, a_{n}\right)$ and $\left(b_{1}, \ldots, b_{n}\right)$ are $n$-tuples of real numbers with $0<m b_{i} \leq a_{i} \leq$ $M b_{i}(1 \leq i \leq n)$, then

- Cassels inequality [49]

$$
\frac{\sum_{k=1}^{n} w_{k} a_{k}^{2} \sum_{k=1}^{n} w_{k} b_{k}^{2}}{\left(\sum_{k=1}^{n} w_{k} a_{k} b_{k}\right)^{2}} \leq \frac{(M+m)^{2}}{4 m M}
$$

- Klamkin-McLenaghan inequality [33]

$$
\sum_{k=1}^{n} w_{k} a_{k}^{2} \sum_{k=1}^{n} w_{k} b_{k}^{2}-\left(\sum_{k=1}^{n} w_{k} a_{k} b_{k}\right)^{2} \leq(\sqrt{M}-\sqrt{m})^{2} \sum_{k=1}^{n} w_{k} a_{k} b_{k} \sum_{k=1}^{n} w_{k} a_{k}^{2}
$$

Now, let $\Gamma$ be a nonempty set and let $\mathcal{L}$ be a linear space of real-valued functions $h: \Gamma \rightarrow \mathbb{R}$ having the property that $e(t)=1(t \in \Gamma)$ belongs to $\mathcal{L}$. A linear functional $\psi$ on $\mathcal{L}$ with $\psi(f) \geq 0$ for $f(t) \geq 0(t \in \Gamma)$ is called an isotonic linear functional. Dragomir [16] gave some generalizations of the C-S inequality. In particular, he showed that if $f, g, f g, f^{2}, g^{2}, f|f|$, $f|g|, g|g|,|f| g$ all belong to $\mathcal{L}$, then for any two isotonic linear functionals $\psi, \tau: \mathcal{L} \rightarrow \mathbb{R}$, one has

$$
\begin{aligned}
\psi\left(f^{2}\right) \tau\left(g^{2}\right) & -2 \psi(f g) \tau(f g)+\psi\left(g^{2}\right) \tau\left(f^{2}\right) \\
& \geq|\psi(f|f|) \tau(g|g|)+\psi(g|g|) \tau(f|f|)-\psi(|f| g) \tau(f|g|)-\psi(f|g|) \tau(|f| g)|
\end{aligned}
$$

Similar results for integrals, isotonic functionals as well as generalizations of reverse C-S inequality in the setting of inner product spaces are well-studied; see e.g. [16]. Zagier [50] showed that if $f, g:[0, \infty) \rightarrow[0, \infty)$ are decreasing functions, then

$\max \left\{f(0) \int_{0}^{\infty} g(t) d t, g(0) \int_{0}^{\infty} f(t) d t\right\} \cdot \int_{0}^{\infty} f(t) g(t) d t \geq\left(\int_{0}^{\infty} f(t)^{2} d t\right)\left(\int_{0}^{\infty} g(t)^{2} d t\right)$. 
Cerone et al. [13] presented a number of reverses of the C-S inequality in the general setting of 2-inner product spaces and an application to integral inequalities in a weighted space.

\section{Operator REVERSE C-S INEqualities}

In the content of $C^{*}$-algebras, Joiţa [30] presented a condition being equivalent to the commutativity of a $C^{*}$-algebra.

Niculescu [41] gave some multiplicative and additive converses of the C-S inequality in the setting of $C^{*}$-algebras. He showed that if $\varphi$ is a positive linear functional on a $C^{*}$-algebra, $\langle C, D\rangle$ is the semi-inner product defined by $\varphi\left(D^{*} C\right), m B \leq A \leq M B$, where $A, B$ are selfadjoint and $m, M$ are positive real numbers, then

$$
\operatorname{Re}\langle A, B\rangle \geq \frac{2 \sqrt{m M}}{m+M}\langle A, A\rangle^{\frac{1}{2}} \cdot\langle B, B\rangle^{\frac{1}{2}}
$$

provided that either $A B=B A$ or $\varphi(C D)=\varphi(D C)$ for all $C, D$ in the $C^{*}$-algebra.

Moslehian and Persson [39] proved other reverse C-S inequalities in the framework of $C^{*}$-algebras and $C^{*}$-modules. See also the books $[26,23]$ and references therein.

In [38] the authors presented a Diaz-Metcalf type operator inequality and applied it to get the operator versions of the Pólya-Szegö, Kantorovich, Shisha-Mond, Cassels and KlamkinMcLenaghan inequalities as some reverse C-S inequalities via a unified approach as follows:

- operator Diaz-Metcalf inequality of first type

$$
M m \Phi(A)+\Phi(B) \leq(M+m) \Phi(A \sharp B) ;
$$

- operator Cassels inequality

$$
\Phi(A) \sharp \Phi(B) \leq \frac{M+m}{2 \sqrt{M m}} \Phi(A \sharp B) ;
$$

- operator Klamkin-McLenaghan inequality

$$
\Phi(A \sharp B)^{\frac{-1}{2}} \Phi(B) \Phi(A \sharp B)^{\frac{-1}{2}}-\Phi(A \sharp B)^{\frac{1}{2}} \Phi(A)^{-1} \Phi(A \sharp B)^{\frac{1}{2}} \leq(\sqrt{M}-\sqrt{m})^{2} ;
$$

- operator Kantorovich inequality

$$
\Phi(A) \sharp \Phi\left(A^{-1}\right) \leq \frac{M^{2}+m^{2}}{2 M m},
$$

where $A, B \in \mathbb{B}(\mathscr{H})$ are positive invertible operators satisfying $m^{2} A \leq B \leq M^{2} A$ for some positive real numbers $m, M$ and $\Phi: \mathbb{B}(\mathscr{H}) \rightarrow \mathbb{B}(\mathscr{K})$ is a positive linear map. They also showed that if the condition $m^{2} A \leq B \leq M^{2} A$ is replaced by $m_{1}^{2} \leq A \leq M_{1}^{2}$ and 
$m_{2}^{2} \leq B \leq M_{2}^{2}$ for some positive real numbers $m_{1}, m_{2}, M_{1}, M_{2}$, then the following inequalities hold instead:

- operator Diaz-Metcalf inequality of second type

$$
\frac{M_{2} m_{2}}{M_{1} m_{1}} \Phi(A)+\Phi(B) \leq\left(\frac{M_{2}}{m_{1}}+\frac{m_{2}}{M_{1}}\right) \Phi(A \sharp B) ;
$$

- operator Pólya-Szegö inequality

$$
\Phi(A) \sharp \Phi(B) \leq \frac{1}{2}\left(\sqrt{\frac{M_{1} M_{2}}{m_{1} m_{2}}}+\sqrt{\frac{m_{1} m_{2}}{M_{1} M_{2}}}\right) \Phi(A \sharp B) ;
$$

- operator Shisha-Mond inequality

$$
\Phi(A \sharp B)^{\frac{-1}{2}} \Phi(B) \Phi(A \sharp B)^{\frac{-1}{2}}-\Phi(A \sharp B)^{\frac{1}{2}} \Phi(A)^{-1} \Phi(A \sharp B)^{\frac{1}{2}} \leq\left(\sqrt{\frac{M_{2}}{m_{1}}}-\sqrt{\frac{m_{2}}{M_{1}}}\right)^{2} ;
$$

These inequalities are indeed the operator version of the corresponding classical inequalities mentioned in the previous section.

One can get the integral versions of discrete reverse inequalities by considering $\mathcal{L}_{\rho}^{2}(\Omega, \mu)$ as a Hilbert space, multiplication operators $\left.A, B \in \mathbb{B}\left(\mathcal{L}_{\rho}^{2}(\Omega, \mu)\right)\right)$ defined by $A(h)=f^{2} h$ and $B(h)=g^{2} h$ for bounded $f, g \in \mathcal{L}_{\rho}^{2}(\Omega, \mu)$ and a positive linear map $\Phi$ by $\Phi(T)=\int_{\Omega} \rho T(1) d \mu$ on $\left.\mathbb{B}\left(\mathcal{L}^{2}(\Omega, \mu)\right)\right)$. For instance, let us state integral versions of the Cassels and KlamkinMcLenaghan inequalities.

Corollary 6.1. [38, Corollary 3.1] Let $f, g \in \mathcal{L}_{\rho}^{2}(\Omega, \mu)$ with $0 \leq m g(t) \leq f(t) \leq M g(t)$ for some positive scalars $m, M$ a.e.. Then

$$
\int_{\Omega} \rho(t) f(t)^{2} d \mu(t) \int_{\Omega} \rho(t) g(t)^{2} d \mu(t) \leq \frac{(M+m)^{2}}{4 M m}\left(\int_{\Omega} \rho(t) f(t) g(t) d \mu(t)\right)^{2}
$$

and

$$
\begin{aligned}
\int_{\Omega} \rho(t) f(t)^{2} d \mu(t) \int_{\Omega} \rho(t) g(t)^{2} d \mu(t) & -\left(\int_{\Omega} \rho(t) f(t) g(t) d \mu(t)\right)^{2} \\
& \leq(\sqrt{M}-\sqrt{m})^{2} \int_{\Omega} \rho(t) f(t) g(t) d \mu(t) \int_{\Omega} \rho(t) f(t)^{2} d \mu(t)
\end{aligned}
$$

If we consider the positive linear functional $\Phi(A)=\sum_{i=1}^{n}\left\langle A x_{i}, x_{i}\right\rangle(A \in \mathbb{B}(\mathscr{H}))$, where $x_{1}, \ldots, x_{n} \in \mathscr{H}$ are fixed vectors, we get the following versions of the Diaz-Metcalf and Pólya-Szegö inequalities in the framework of Hilbert spaces. 
Corollary 6.2. [38, Corollary 3.2] Let $\mathscr{H}$ be a Hilbert space, let $x_{1}, \ldots, x_{n} \in \mathscr{H}$ and let $A, B \in \mathbb{B}(\mathscr{H})$ be positive operators satisfying $0<m_{1} \leq A \leq M_{1}$ and $0<m_{2} \leq B \leq M_{2}$. Then

$$
\frac{M_{2} m_{2}}{M_{1} m_{1}} \sum_{i=1}^{n}\left\|A x_{i}\right\|^{2}+\sum_{i=1}^{n}\left\|B x_{i}\right\|^{2} \leq\left(\frac{M_{2}}{m_{1}}+\frac{m_{2}}{M_{1}}\right) \sum_{i=1}^{n}\left\|\left(A^{2} \sharp B^{2}\right)^{1 / 2} x_{i}\right\|^{2}
$$

and

$$
\begin{aligned}
\left(\sum_{i=1}^{n}\left\|A x_{i}\right\|^{2}\right)^{1 / 2}\left(\sum_{i=1}^{n}\left\|B x_{i}\right\|^{2}\right)^{1 / 2} & \\
& \leq \frac{1}{2}\left(\sqrt{\frac{M_{1} M_{2}}{m_{1} m_{2}}}+\sqrt{\frac{m_{1} m_{2}}{M_{1} M_{2}}}\right) \sum_{i=1}^{n}\left\|\left(A^{2} \sharp B^{2}\right)^{1 / 2} x_{i}\right\|^{2} .
\end{aligned}
$$

An inequality complementary to the C-S inequality is given by Lee [35]. She showed that if $\Phi$ is a positive linear map and $A, B$ are positive definite matrices such that $m A \leq B \leq M A$ for some positive real numbers $m, M$, then

$$
\Phi(A) \sharp \Phi(B) \leq \frac{(M / m)^{1 / 4}+(m / M)^{1 / 4}}{2} \Phi(A \sharp B) .
$$

For a fixed orthonormal basis $\left\{e_{n}\right\}$ of a separable Hilbert space $\mathscr{H}$, the Hadamard (or Schur) product $A \circ B$ of two bounded operators $A$ and $B$ acting on $\mathscr{H}$ is defined by

$$
\left\langle(A \circ B) e_{i}, e_{j}\right\rangle=\left\langle A e_{i}, e_{j}\right\rangle\left\langle B e_{i}, e_{j}\right\rangle
$$

There are some C-S inequalities for Hadamard product. The following inequality is due to Ando [4]

$$
A \circ B \leq\left(A^{2} \circ I\right)^{1 / 2}\left(B^{2} \circ I\right)^{1 / 2} \quad(A, B \geq 0)
$$

and another is proved by Aujla and Vasudeva [6]

$$
A \circ B \leq\left(A^{2} \circ B^{2}\right)^{1 / 2} \quad(A, B \geq 0) .
$$

Horn and Mathias [43] proved the following C-S type inequalities for $n \times n$ complex matrices $A, B$, the inequalities $\left\|A^{*} B\right\|^{2} \leq\left\|A^{*} A\right\|\left\|B^{*} B\right\|$ and $\|A \circ B\|^{2} \leq\left\|A^{*} A\right\|\left\|B^{*} B\right\|$ hold.

\section{Operator Wielandt inEQUAlity}

In this section, we pay attention to the Wielandt inequality [27, 7.4.32], an improvement of the C-S inequality,

$$
|\langle A y, x\rangle|^{2} \leq\left(\frac{M-m}{M+m}\right)^{2}\langle A x, x\rangle\langle A y, y\rangle
$$


where $A$ is a positive operator with $m \leq A \leq M$ for some positive real numbers $m, M$ and $x, y$ are orthogonal vectors.

In accordance with [21], we pose two proofs of the Wielandt inequality.

The first one is inspired by that of C-S inequality, in which the discriminant is used.

Proof I. It follows from $m \leq A \leq M$ that for all complex numbers $\lambda$,

$$
m\|x+\lambda y\|^{2} \leq\langle A(x+\lambda y), x+\lambda y\rangle \leq M\|x+\lambda y\|^{2} .
$$

Without loss of generality we may assume that $\langle A y, x\rangle \geq 0$. We have

$$
\begin{gathered}
(\langle A y, y\rangle-m) t^{2}+2\langle A y, x\rangle t+\langle A x, x\rangle-m \geq 0, \quad \text { and } \\
(M-\langle A y, y\rangle) t^{2}+2\langle A y, x\rangle t+M-\langle A x, x\rangle \geq 0
\end{gathered}
$$

for all real numbers $t$. By (first) $\times M+($ second $) \times m$, we observe that

$$
(M-m)\langle A y, y\rangle t^{2}+2(M+m)\langle A y, x\rangle t+(M-m)\langle A x, x\rangle \geq 0 \quad(t \in \mathbb{R}),
$$

or equivalently,

$$
(M+m)^{2}\langle A y, x\rangle^{2} \leq(M-m)^{2}\langle A x, x\rangle\langle A y, y\rangle
$$

which implies (7.1).

Another proof is along with [7.4.26] in Horn-Johnson's textbook [27].

Proof II. Set

$$
C=\left(\begin{array}{ll}
\langle A x, x\rangle & \langle A y, x\rangle \\
\langle x, A y\rangle & \langle A y, y\rangle
\end{array}\right)
$$

Then $m \leq C \leq M$ since for any unit vector $z={ }^{t}(\alpha, \beta) \in \mathbb{C}^{2},\|\alpha x+\beta y\|=\|z\|=1$ and $\langle C z, z\rangle=\langle A(\alpha x+\beta y), \alpha x+\beta y\rangle \in[m, M]$. So the spectrum $\sigma(C)=\{a, b\} \subseteq[m, M]$. Since

$$
1-\frac{|\langle A y, x\rangle|^{2}}{\langle A x, x\rangle\langle A y, y\rangle}=\frac{4 \operatorname{det} C}{(\operatorname{tr} C)^{2}-(\langle A x, x\rangle-\langle A y, y\rangle)^{2}} \geq \frac{4 \operatorname{det} C}{(\operatorname{tr} C)^{2}}=\frac{4 a b}{(a+b)^{2}},
$$

we have

$$
\frac{|\langle A y, x\rangle|^{2}}{\langle A x, x\rangle\langle A y, y\rangle} \leq 1-\frac{4 a b}{(a+b)^{2}}=\left(\frac{1-\frac{b}{a}}{1+\frac{b}{a}}\right)^{2} \leq\left(\frac{1-\frac{M}{m}}{1+\frac{M}{m}}\right)^{2}=\left(\frac{M-m}{M+m}\right)^{2}
$$

by the monotonicity of the function $\frac{t-1}{t+1}$.

The Wielandt inequality was generalized by Bauer and Householder, see e.g. [7, Theorem II]:

$$
|\langle A y, x\rangle|^{2} \leq\left(\frac{M_{0}-m_{0}}{M_{0}+m_{0}}\right)^{2}\langle A x, x\rangle\langle A y, y\rangle
$$


$A$ is a positive operator satisfying $m \leq A \leq M$ for some positive real numbers $m, M, x, y$ are unit vectors, $M_{0}=M(1+|\langle x, y\rangle|)$ and $m_{0}=m(1-|\langle x, y\rangle|)$. This is called Bauer-Householder inequality.

The second proof is generalized in order to correspond to the Bauer-Householder inequality.

Lemma 7.1. If $A$ satisfies $m \leq A \leq M$ for some positive real numbers $m, M$ and

$$
C=\left(\begin{array}{ll}
\langle A x, x\rangle & \langle A y, x\rangle \\
\langle x, A y\rangle & \langle A y, y\rangle
\end{array}\right)
$$

for given unit vectors $x, y$. Then $m_{0} \leq C \leq M_{0}$, where $M_{0}=M(1+|\langle x, y\rangle|)$ and $m_{0}=$ $m(1-|\langle x, y\rangle|)$.

Proof. We take $X=[x, y]$ of $\mathbb{C}^{2}$ into $\mathscr{H}$, i.e., $[x, y]^{t}(\alpha \beta)=\alpha x+\beta y$. Then we have $C=X^{*} A X$ and $W^{-}\left(X^{*} X\right)=c o \sigma\left(X^{*} X\right)=[1-t, 1+t]$, where $W^{-}(Y)$ is the closed numerical range of $Y$ and $t=|\langle x, y\rangle|$. Hence it follows that

$$
\sigma(C) \subseteq W^{-}(C)=W^{-}\left(X^{*} A X\right) \subseteq W^{-}(A) W^{-}\left(X^{*} X\right) \subseteq[m, M][1-t, 1+t]=\left[m_{0}, M_{0}\right]
$$

By Lemma 7.1, we have a simple proof of the Bauer-Householder inequality. As a matter of fact, as in the second proof,

$$
\frac{|\langle A y, x\rangle|^{2}}{\langle A x, x\rangle\langle A y, y\rangle} \leq 1-\frac{4 a b}{(a+b)^{2}}=\left(\frac{1-\frac{b}{a}}{1+\frac{b}{a}}\right)^{2}
$$

for $a, b$ with $\{a, b\}=\sigma(C)$. Since $\sigma(C) \subseteq\left[m_{0}, M_{0}\right]$, we have the desired inclusion.

Next, using a similar argument as in the first proof of the Wielandt inequality, we have the following more general inequality.

Theorem 7.2. If $A$ satisfies $m \leq A \leq M$ for some positive real numbers $m$, $M$, then

$$
|\langle A y, x\rangle| \leq \frac{M-m}{M+m}\langle A x, x\rangle^{\frac{1}{2}}\langle A y, y\rangle^{\frac{1}{2}}+\frac{2 M m}{M+m}|\langle x, y\rangle| .
$$

The extension of the Heinz-Kato inequality by Furuta in [25] is called now the HeinzKato-Furuta inequality. Wielandt type inequalities associated to the Heinz-Kato-Furuta inequality are given in [24]. In the next corollary we present an equivalent inequality to (7.2). 
Corollary 7.3. $T \in \mathbb{B}(\mathscr{H})$ satisfying $m \leq T \leq M$ for some positive real numbers $m, M$, Then for each $\gamma>0$

$$
\left|\left\langle T|T|^{\alpha+\beta-1} y, x\right\rangle\right| \leq \frac{M^{\gamma}-m^{\gamma}}{M^{\gamma}+m^{\gamma}}\left\|\left.T\right|^{\alpha} y\right\|\left\|\left|T^{*}\right|^{\beta} x\right\|+\frac{2 M^{\gamma} m^{\gamma}}{M^{\gamma}+m^{\gamma}}\left|\left\langle T|T|^{\alpha+\beta-\gamma-1} y, x\right\rangle\right|
$$

holds for $x, y \in H$ and $\alpha, \beta \in \mathbb{R}$.

Proof. Let $T=U|T|$ be the polar decomposition of $T$. For given $x, y \in H$, we put $x_{1}=$ $|T|^{\beta-\frac{\gamma}{2}} U^{*} x$ and $y_{1}=|T|^{\alpha-\frac{\gamma}{2}} y$. Since $0<m^{\gamma} \leq|T|^{\gamma} \leq M^{\gamma}$ and $U|T|^{\beta} U^{*}=\left|T^{*}\right|^{\beta}$, we have the conclusion by applying Theorem 7.2 to $x_{1}, y_{1}$ and $A=|T|^{\gamma}$.

If we take two real numbers $\alpha, \beta$ with $\alpha+\beta=1$ and $\gamma=1$ in the above corollary, we reach another equivalent inequality to (7.2).

Corollary 7.4. Let $T \in \mathbb{B}(\mathscr{H})$ satisfying $m \leq T \leq M$ for some positive real numbers $m, M$. Then

$$
|\langle T y, x\rangle| \leq \frac{M-m}{M+m}\left\||T|^{\alpha} y\right\|\left\|\left.|| T^{*}\right|^{\beta} x\right\|+\frac{2 M m}{M+m}\left|\left\langle T|T|^{-1} y, x\right\rangle\right|
$$

holds for $x, y \in H$ and $\alpha, \beta \in \mathbb{R}$.

We conclude this section with a discussion on relations among the C-S, Wielandt and Kantorovich inequalities.

For given unit vectors $x, y$, we put $v=y-\langle y, x\rangle x$. Since $\langle v, x\rangle=0$, we get

$$
|\langle A v, x\rangle|^{2} \leq K\langle A x, x\rangle\langle A v, v\rangle,
$$

where $K=\left(\frac{M-m}{M+m}\right)^{2}$. The latter inequality is equivalent to

$$
|\langle A y, x\rangle|^{2} \leq\langle A x, x\rangle\langle A y, y\rangle-\left(\frac{1}{K}-1\right)|\langle y, x\rangle\langle A x, x\rangle-\langle A y, x\rangle|^{2},
$$

which clearly improves C-S inequality.

If we take $y=A^{-1} x$ for a unit vector $x$ in the above inequality, we obtain the Kantorovich inequality,

$$
\langle A x, x\rangle\left\langle A^{-1} x, x\right\rangle \leq \frac{(M+m)^{2}}{4 M m} \quad \text { if } M \geq A \geq m>0
$$

Acknowledgement. The first named author was partially supported by grant MTM201237436-C02-02 from the MINECO of Spain. The fourth named author would like to thank the Department of Mathematics and Computer Science at Karlstad University for their kind hospitality during his stay there. 


\section{REFERENCES}

1. J.M. Aldaz, A stability version of Hölder's inequality, J. Math. Anal. Appl. 343 (2008), no. 2, 842-852.

2. J.M. Aldaz, Strengthened Cauchy-Schwarz and Hölder inequalities, J. Inequal. Pure Appl. Math. 10 (2009), no. 4, Article 116, 6 pp.

3. H. Alzer, On the Cauchy-Schwarz inequality, J. Math. Anal. Appl. 234 (1999), no. 1, 6-14.

4. T. Ando, Topics on operator inequality, Hokkaido Univ. Lecture Note, 1978.

5. Lj. Arambasić, D. Bakić and M.S. Moslehian, A treatment of the Cauchy-Schwarz inequality in $C^{*}$ modules, J. Math. Anal. Appl. 381 (2011) 546-556.

6. J.S. Aujla and H.L. Vasudeva, Inequalities involving Hadamard product and operator means, Math. Japon. 42 (1995), no. 2, 265-272.

7. F.L. Bauer and A.S. Householder, Some inequalities involving the euclidean condition of a matrix, Numer. Math. 2 (1960), 308-311

8. R. Bhatia and C. Davis, A Cauchy-Schwartz inequality for operators with applications Linear Algebra Appl. 223/224 (1995), 119-129.

9. R. Bhatia and C. Davis, More operator versions of the Schwarz inequality, Comm. Math. Phys. 215 (2000), no. 2, 239-244.

10. V. Bouniakowsky, Sur quelques inegalités concernant les intégrales aux différences finies, Mem. Acad. Sci. St. Petersbourg 7 (1859), no. 1, p. 9.

11. M.L. Buzano, Generalizzatione della diseguagliazza di Cauchy?Schwarz, Rend. Sem. Mat. Univ. Politech. Torino 31 (1971/73) 405-?409.

12. A. Cauchy, Oeuvres 2, III (1821), p. 373.

13. P. Cerone, Y.J. Cho, S.S. Dragomir and S.S. Kim, Refinements of some reverses of Schwarz's inequality in 2-inner product spaces and applications for integrals, J. Indones. Math. Soc. 12 (2006), no. 2, 185-199.

14. M.-D. Choi, A Schwartz inequality for positive linear maps on $C^{*}$-algebras, Illinois J. Math. 18 (1974), $565-574$.

15. J.B. Diaz and F.T. Metcalf, Stronger forms of a class of inequalities of G. Pólya-G. Szegö and L.V. Kantorovich, Bull. Amer. Math. Soc. 69 (1963), 415-418.

16. S.S. Dragomir, A survey on Cauchy-Bunyakovsky-Schwarz type discrete inequalities, J. Inequal. Pure Appl. Math. 4 (2003), no. 3, Article 63, 142 pp.

17. S.S. Dragomir, Advances in inequalities of the Schwarz, triangle and Heisenberg type in inner product spaces, Nova Science Publishers, Inc., New York, 2007.

18. N. Elezović, Lj. Marangunić and J.E. Pečarić, Unified treatment of complemented Schwarz and Grüss inequalities in inner product spaces, Math. Inequal. Appl. 8 (2005), no.2, 223-231.

19. J.I. Fujii, Operator-valued inner product and operator inequalities, Banach J. Math. Anal. 2 (2008), no. $2,59-67$.

20. J.I. Fujii, M. Fujii, M.S. Moslehian and Y. Seo, Cauchy-Schwarz inequality in semi-inner product $C^{*}$ modules via polar decomposition, J. Math. Anal. Appl. 394 (2012), no. 2, 835-840.

21. M. Fujii, Y. Katayama and R. Nakamoto, Generalizations of the Wielandt theorem, Math. Japon. 49 (1999) 217-222. 
22. M. Fujii, S. Izumino, R. Nakamoto and Y. Seo, Operator inequalities related to Cauchy-Schwarz and Holder-McCarthy inequalities, Nihonkai Math. J. 8 (1997) 117-122.

23. M. Fujii, J. Mićić Hot, J. Pečarić and Y. Seo, Recent Developments of Mond-Pečarić Method in Operator Inequalities, Inequalities for bounded selfadjoint operators on a Hilbert space, II, Element, Zagreb, 2012.

24. M. Fujii and Y. Seo, Wielandt type extensions of the Heinz-Kato-Furuta inequality, Operator Theory: Adv. Appl. 127 (2001), 267-277.

25. T. Furuta, An extension of the Heinz-Kato theorem, Proc. Amer. Math. Soc. 120 (1994), 785-787.

26. T. Furuta, J. Mićić Hot, J. Pečarić and Y. Seo, Mond-Pečarić Method in Operator Inequalities, Element, Zagreb, 2005.

27. R.A. Horn and C.R. Johnson, Matrix Analysis, Cambridge University Press, Cambridge, 1985.

28. D. Ilišević and S. Varošanec, On the Cauchy-Schwarz inequality and its reverse in semi-inner product $C^{*}$-modules, Banach J. Math. Anal. 1 (2007), 78-84.

29. D.R. Jocić, Cauchy-Schwarz and means inequalities for elementary operators into norm ideals, Proc. Amer. Math. Soc. 126 (1998), no. 9, 2705-2711.

30. M. Joiţa, On the Cauchy-Schwarz inequality in $C^{*}$-algebras, Math. Rep. (Bucur.) 3(53) (2001), no. 3, 243-246.

31. R.V. Kadison, A generalized Schwarz inequality and algebraic invariants for operator algebras, Ann. of Math. (2) 56 (1952), 494-503.

32. F. Kittaneh, Some norm inequalities for operators, Canad. Math. Bull. 42 (1999), no. 1, 87-96.

33. M.S. Klamkin and R.G. Mclenaghan, An ellipse inequality, Math. Mag. 50 (1977), 261-263.

34. E.C. Lance, Hilbert $C^{*}$-modules, A Toolkit for Operator Algebraists, London Math. Soc. Lecture Series, 210. Cambridge University Press, Cambridge, 1995.

35. E.-Y. Lee, A matrix reverse Cauchy-Schwarz inequality, Linear Algebra Appl. 430 (2009), no. 2-3, $805-810$.

36. J. Ma, An identity in real inner product space, J. Inequal. Pure Appl. Math. 8 (2007), no. 2, Art. 48, 4 pp.

37. R. Mathias, A note on: "More operator versions of the Schwarz inequality" [Comm. Math. Phys. 215 (2000), no. 2, 239-244; by R. Bhatia and C. Davis], Positivity 8 (2004), no. 1, 85-87.

38. M.S. Moslehian, R. Nakamoto and Y. Seo, A Diaz-Metcalf type inequality for positive linear maps and its applications, Electron. J. Linear Algebra 22 (2011), 179-190.

39. M.S. Moslehian and L.-E. Persson, Reverse Cauchy-Schwarz inequalities for positive $C^{*}$-valued sesquilinear forms, Math. Inequal. Appl. 12 (2009), no. 4, 701-709.

40. R.B. Nelsen, Proof without Words: The Cauchy-Schwarz Inequality, Math. Mag. 67 (1994), no. 1, 20.

41. C.P. Niculescu, Converses of the Cauchy-Schwarz inequality in the $C^{*}$-framework, An. Univ. Craiova Ser. Mat. Inform. 26 (1999), 22-28.

42. G. Pólya and G. Szegö, Aufgaben und Lehrsätze aus der Analysis, Vol. 1, Berlin 1925, pp. 57 and $213-214$.

43. R.A. Horn and R. Mathias, An analog of the Cauchy-Schwarz inequality for Hadamard products and unitarily invariant norms, SIAM J. Matrix Anal. Appl. 11 (1990), no. 4, 481-498. 
44. O. Shisha and B. Mond, Bounds on differences of means, Inequalities I, New York-London, 1967, 293308.

45. J.M. Steele, The Cauchy-Schwarz master class. An introduction to the art of mathematical inequalities, MAA Problem Books Series. Mathematical Association of America, Washington, DC; Cambridge University Press, Cambridge, 2004.

46. W.F. Stinespring, Positive functions on $C^{*}$-algebras, Proc. Amer. Math. Sci. 6 (1955), 211-216.

47. S. Wada, On some refinement of the Cauchy-Schwarz inequality, Linear Algebra Appl. 420 (2007), no. 2-3, 433-440.

48. S.S. Wagner, Notices Amer. Math. Soc. 12 (1965), 220.

49. G.S. Watson, Serial correlation in regression analysis I, Biometrika 42 (1955), 327-342.

50. D.B. Zagier, An inequality converse to that of Cauchy, Indag. Math. (N.S.) 39 (1977), no. 4, 349-351.

${ }^{1}$ ICMAT and Departamento de Matemáticas, Universidad Autónoma de Madrid, CantoBLANCO 28049, Madrid, SPAin.

E-mail address: jesus.munarriz@uam.es

2 Department of Mathematics and Computer Science, Karlstad University, SE-65188 KARLSTAD, SWEDEN.

E-mail address: sorina.barza@kau.se

${ }^{3}$ Department of mathematics, Osaka Kyoiku University, Asahigaoka, Kashiwara, Osaka 582-8582, JAPAN

E-mail address: mfujii@cc.osaka-kyoiku.ac.jp

${ }^{4}$ Department of Pure Mathematics, Center of Excellence in Analysis on Algebraic Structures (CEAAS), Ferdowsi University of Mashhad, P.O. Box 1159, Mashhad 91775, Iran; Department of Mathematics and Computer Science, Karlstad University, SE-65188 KarlSTAD, SWEDEN.

E-mail address: moslehian@um.ac.ir and moslehian@member.ams.org 